in any other way. Now, when such a man as Dr. T. A. Emmet could say that for a year he had seen in his large practice only one case of disease of the tubes, and that the patient retused to be operated upon, and got well in a few months, it seemed very strange for him (the speaker) to say that he found from twenty to thirty cases every year. He would explain this by saying that Dr. Emmet and he differed in their views as to the pathological conditions to be found in the pelvis of the same woman; and the reason he got so many cases was not that he found them all in his own private practice, but that cases were sent to him by other physicians. He could not go so far as Dr. Gordon in operating on cases for subjective symptoms alone. In all his cases, except five or six, he could make out by a local examination that the appendages were diseased.

Dr. Divies said that the removal of ovaries for the cure of various neurstic conditions gave no help. He had seen them removed in two cases for the cure of epilepsy (not in Dr. Imlach's practice) without benefit. With regard to the tubes, their pathology should be studied in the fresh state, as, after preserving in spirit, etc., no adequate idea could be formed of their former condition. He believed that there was no possible means of treating these diseases except by operation.

Dr. H. P. C. WILson (Baltimore) held that the operation was a justifiable one in suitable cases.

Mr. LAWSON TAIT said that the turn of the discussion being wholly in the direction of approval, made it necessary for him to say but little. With what Dr. Savage had said, he entirely concurred. Dr. Savage and he had worked together in friendly rivalry for fifteen years, seeing each other's work with an equally earnest desire to arrive at accurate conclusions, correcting in all they could what was faulty, and approving what was right; and when two men could do that as colleagues, they achieved a position which it was extremely difficult to overturn. There kad been much misrepresentation, partly wilful, and partly arising from ignorance. One instance of misrepresentation was the use of the word "spaying." He was amazed that so philosophical a nation as the Germans should conticue to use the term "cas. tration." The operation of spaying animals was performed before the onset of puberty. Who had ever removed the uterine appendages in a human child? Spaying was performed for the purpose of keep. ing the animal quiet, and improving the food-supply. No such object was sought in the operations on women, nothing but the saving of lif $\theta$ and the arrest of suffering. Spaying an animal and the surgical operation of removal of the uterine appendages in a woman had, therefore, no concoivable resemblance, not even in the details of the operation, and the use of the word was a misrepresentation, based on the densest and most inexcusable ignorance. Another misrepresentation was as to the frequency of the operation. His eminent fellow-townsman, Mr. Vose Solomon, who sat by, nperated on large numbers of cases of cataract, and he (Mr. Tait) operated on none. He charged fir. Solomon with being guilty of having a great ophthalmic reputation. His friend, Dr. Davidson of Liverpool, was an eminent consulting plyssician, and he (MIr. Tait) deliberately charged Dr. Davidson with receiving a large number of cases of pneumonia. Dr. Davidson would probably $\epsilon$ xcuse himself by the plea that his position and reputation brought him inevitably into the position of being obliged to plead guilty to the charge. Concerning disease of the uterine appendages, Mr. Tait had to say that this was precisely his own position.

Dr. H. A. KELLy said there certainly existed at the present time an epidemic zeal for operations upon tubes and ovaries, and it was time for bodies like the present, and the American Gynæcological Society, to enter protests, and lay down clearly-defined rules for the guidance of those practitioners coming within the range of their influence. In a code of such rules, one of prime importance was the following: that all operators, without exception, should preserve their specimens. Dr. Kelly stated that the fear of making a long incision was, in his opinion, due to certain deductions made by Sir Spencer Wells from his own experience with large ovarian tumours, where the length of the incision was-as was demonstrable from Sir Spencer Wells's own table-dangerous not per se, but simply as an index of the gravity of the operation in other respects. Therefore, the rule should be to make the incision long enough to allow of working with facility, and completing the operation within the shortest time possible.

Dr. Graily HewitT considered the profession under a debt of gratitude to those who had performed these operations for information which had proved valuable. Still, far too little was yet known to allow of generalisations of a complete kind. In regard to chronic disease of incurable character-the incentive to operation in some cases -he believed that the difficulty was associated with nutritional defects, and that cure could be attained by elevating the nutritional condition by rest, massage, nursing, and good feeding. One great difficulty was connected with the diagnosis. That should be made more complete by careful double examination. He considered chronic displacements were too often treated by this operation. In some such, the operation was in exceptional cases admissible. Displaced ovaries could be otherwise easily cured, a well-adjusted soft Hodge's pessary being used. Gonorrhœa had been shown to be more productive of internal disease than had been supposed, but he thought its frequency was overestimated.

Dr. MORE MADDEN said that, whilst the previous speakers had been almost unanimous in condemning his heterodoxy on the subject under discussion, they had differed so widely among themselves as to almost every other point as to have left little for him to answer. Removal of the uterine appendages might be useful for fibromata, but he was pleased that Dr. Bantock had adopted his views as to the enucleation of such tumours in suitable cases. In spite of the eloquent speeches to which he had listened, he "was of the same opinion still" regarding his contention that laparotomy was performed too frequently and in unsuitable cases. In that he hoped that he was neither prejudiced nor ignorant, as he had been said to be.

\section{RECENT OBSERVATIONS ON URINE-TESTING.}

BY GEORGE JOHNSON, M.D., F.R.S.,

Emeritus Professor of Clinical Medicine; Consulting Physician to King's College Hospital.

THE nature of the material in normal urine which resembles glucose in its reducing action upon cupric oxide and upon picric acid has often been the subject of inquiry and discussion. In a paper published in the MLedico-Chirurgical Transactions, vol. lxviii, p. 222, Dr. Pavy writes as follows: "The reducing action [on cupric oxide], before the addition of acetate of lead, is due partly to uric acid, and partly to the small amount of sugar naturally present in urine. It is doubtful if there is any other body worthy of consideration to exert any sensible reducing effect."

Dr. Pavy ascertained that one-fourth of the reducing action of normal urine upon copper oxide is removed after the precipitation of the uric acid by lead acetate, and the remaining three-fourths he attributes to sugar.

In my Lectures on Albumen and Sugar Testing, p. 43 (Messrs. Smith, Elder, and Co.), I have shown that all normal urines yield, when tested by boiling with picric acid and potash, a depth of colour which, if glucose were the reducing agent, would indicate, upon an average, 0.6 grain per ounce; the amount usually varying from 0.5 to 0.7 , and sometimes, in a concentrated specimen of high specific gravity, amounting to one grain per ounce.

At the time of publication of those lectures, I expressed my belief that, if the reducing substance were not glucose, "it must surely be some nearly allied substance" (p. 44). Since then, my son has been engaged in an elaborate investigation of the reducing agent which is always present in normal urine. The results he will shortly publish in detail. Meanwhile, I may state that he has conclusively proved : 1. That not a trace of glucose is to be found in normal urine; 2 . That, as uric acid has no reducing action on picric acid, the chief, if not the sole, constituent of normal urine which reduces picric acid in the presence of potash is kreatinine. He has discovered a simple means of precipitating kreatinine from the urine in combination with a metallic salt, and by a process which has no effect upon any glucose that may be present. A specimen of normal urine which has been thus treated ceases to give the red colour when boiled with picric acid and potash, but if, before the separation of the kreatinine, glucose be added to the urine in the proportion of a grain to the ounce, the sugar is found undiminished after the kreatinive has been removed.

Urine of Paticnts who are taking Salicylate of Soda.-I have often found that the urine of patients who are taking salicylate of soda, when boiled with picric acid and potash, gives a deeper red colour than is quite normal, the colour being equal to that which would result from the presence of from one to two grains of glucose per ounce. Fehling's solution is also reduced, as it would be by a small amount of glucose. My son has for some time been investigating the nature of this reducing agent. $\mathrm{He}$ has ascertained that it is neither glucose nor an excess of kreatinine. It is probably due to some product metabolism of the salicylate of soda, the precise nature of which $\mathbf{r}$ mains to be ascertained. The salt itself has no reducing action $o$ either picric acid or cupric oxide.

My reason for referring to the subject now is to indicate that th increased reducing action on picric acid and copper oxide of the urin 
from patients who are taking salicylate of soda is not to be attrihuted to the presence of glucose in small quantity.

The Reducing Action of Chloral-Hydrate upon Cupric Oxide.-Dr. Sherwin (Boston Med. Journ., November 18th, 1886, p. 487, and Pharmaceutical Journal, December 25 th, 1886, p. 507) states that a sample of urine, specific gravity 1015, to which a few grains of chloral-hydrate had been added to prevent putrefaction, reduced alkaline copper solution like diabetic urine. He also found that a very weak solution of chloral-hydrate in water reduced Fehling's solution, and the urine of two patients who had taken a medicinal dose of chloral gave a similar reaction. I have repeated and confirmed $\mathrm{Dr}$. Sherwin's observations, and I have further ascertained that, while chloral-hydrate has a powerful reducing action on the copper solution, it has none upon the picric acid test.

The Potassio-Mercuric Todide Test for Albumen is Fallacious. -The Albumen Tests Committee of the Clinical Society, in their report, published in the last volume (xix) of the Transactions, state : "That a solution of potassio-mercuric iodide with citric acid, particularly when used after Heller's method, gives the most delicate and clearly marked reaction." It is very noteworthy that the committee make no mention of the fact which has often been recorded, and which must now be pretty generally known, that this test causes an opalescence, not to be distinguished from a slight albuminous precipitate, in every normal urine. This reagent apparently precipitates, not only mucin, but also some other ingredient of normal urine. For it is found that when the mucin in a normal specimen has been precipitated by citric or acetic acid, and then separated by filtration, the addition of the potassio-mercuric iodide to the clear urine causes a further precipitation and opalescence. It is obvious, therefore, that this reagent, if relied upon as a test for a minute trace of albumen, would be seriously misleading.

The Quantitative Estimate of Albumen. - Since attention has been drawn to the use of Esbach's graduated tubes by Dr. Veale (BritisH Medical Journal, May, 1884), by Mr. Blomfield (Lancet, January 23rd, 1886), and by Dr. Sidney Coupland and myself (Lancet, July, 1886, p. 63), this simple and accurate method of ascertaining the actual amount of albumen has been coming into general use. $\mathrm{Mr}$. Cetti, of 36 , Brook Street, Holborn, who supplies the tubes, with printed directions for their use, at a small cost, tells me that there is a constant and increasing demand for them. The method combines simplicity and facility of working with accuracy of results in a quite remarkable degree.

The most convenient Pocket Test Case. - After trying a great variety of pocket test cases, I have found none so convenient as the "nickelplated pocket urine test case," made by Ernst Jahncke, Canonbury Works, Dorset Street, Essex Road, N., and sold by most surgical instrument makers and nharmaceutical chemists. The case admits of some variety in its fittings and contents. In my own case, which, after two years' use, is as bright and clean as when it was new, I nave two test tubes one within the other, and within the smaller tube a nipple pipette, which is very useful for transferring the urine to the test tube. Then there is a small spirit-lamp, the glass cap of which is so accurately fitted that no spirit leaks out, a stoppered bottle for powdered picric acid, another for grain pellets of caustic potash, and a drachm measure. There are also some blue and red litmus papers.

The case, therefore, contains all the materials required for bedside albumen and sugar testing. In testing for albumen, as much picric acid powder as can be carried on the end of a penknife (about a grain by weight) is dissolved in about a drachm of water by the aid of the lamp. To this is added an equal volume of urine, when the presence of albumen is at once made apparent. Then, to test for sugar, a grain pellet of potash is added to the mixed urine and picric acid, and the liquid is again boiled for a few seconds. If sugar is present, the liquid will be rendered so dark, that no red colour is visible through the test-tube. By diluting the urine in a definite proportion until the standard red colour is obtained, a proximate estimate of the proportion of sugar may be obtained, but, for an accurate analysis, the picro-saccharometer must be used.

The Albumen Tests Committee admit that the possibility of being able to test for both albumen and sugar, by picric acid, with the addition of potash, "renders this very portable test most useful ;" but they add, "There is, however, some practical difficulty here arising out of the hygroscopic quality of the potash cubes recommended by Dr. Johnson." I can assure the committee, and all whom it may concern, that there is here no practical difficulty whatsoever. I have some potash pellets which were supplied to me six months ago by Mr. W. T. Cooper, of 66, Oxford Street, and they are as dry and hard now as when I first received them. Doubtless caustic potash exposed to the air will deliquesce, but, to prevent this, nothing more is re- quired than to keep it in a bottle, the glass stopper of which is smeared with vaseline. This effectually excludes air and moisture, and also prevents the stopper from becoming fixed by the corrosive alkali.

\section{PERIPHERAL NEURITIS IN RAYNAUD'S DISEASE (SYMMETRICAL GANGRENE). ${ }^{1}$}

\section{BY JOSEPH WIGLESWORTH, M.D.LOND.,} Assistant Medical Oflicer, Rainhill Asylum.

THIs communication had reference to a case of Raynaud's disease, under the observation of the author, in Rainbill Asylum, in which changes in the direction of chronic inflammation or sclerosis were found in the peripheral nerves after death.

The patient, a married woman, aged 26 , was admitted from the workhouse December 27th, 1884, and died May 10th, 1886. She suffered from epilepsy, the monthly average of fits being about $2 \frac{1}{2}$. Her mental conaition was one of mild dementia; but she occasionally had slight, transient, maniacal attacks, and exhibited at times hallucinations of hearing. Physically, she was a small, thin, badlydeveloped young woman, measuring 4 feet $10 \frac{1}{2}$ inches in height, and weighing only 79 pounds. There was a peculiar macular eruption scattered over face, trunk, and extremities, but most marked on the face, consisting of irregular blotches of a rather deep port-wine colour; these blotches faded completeily under pressure, and were apparently caused by stasis in the capillaries; they underwent no change with time.

There was slight bronchitis; the heart was distinctly hypertrophied; and the urine contained permanently about one-fourth albumen. Both hands were very small and slender, the muscles were everywhere atrophied, and several fingers had undergone partial amputation. Thus, in the right hand, the thumb, first, and third fingers consisted of little more than the first phalanx, while the second finger consisted of this phalanx only, the fourth finger alone being entire. In the left hand, tho second finger was reduced to the first phalinx, whilst the other fingers were entire, but contracted in flexion. The right foot had lost the big toe from amputation, and in the left foot the corresponding toe had undergone considerable loss of substance, and presented an unhealed sore. The patient herself stated, with reference to these losses of substance, that the parts mortified and were removed by a doctor-which was, without doubt, just what occurred ; for, not only did the patient's mental condition permit of reliance being placed on her statements, but the appearance of the hands was quite characteristic.

Actual gangrene was observed whilst the pationt was under observation. She suffered at irregular intervals from small excoriations, which formed chiefly on the tips of the fingers, or on the extensor aspects of the fingers and toes; at one time a superficial slough formed over the right olecranon, on the separation of which a sore was left about the size of a shilling. But the most marked example of gangrene was the formation of a slough on the extensor aspect of the second phalangeal joint of the left fourth finger; this rapidly spread upwards and downwards, opened into the second phalangeal joint, and necessitated amputation at the joint below. The patient's death occurred rather suddenly, after an epileptic fit, on May 10th, 1886. At the necropsy, thirteen hours after death, the brain weighed 1,176 grammes, and, with the exception of slight thickeving of the membranes and slight wasting of the gyri, appeared quite normal. The lungs were moderately congested, and the heart (weight 9 ounces) showed considerable hypertrophy of the left ventricle. The liver and spleen were normal, but the kidneys (right $2 \frac{1}{2}$ ounces, left 23 ounces) were diminished in size, with adherent capsules, granular surfaces, and mottled cortices, containing numerous cysts.

Raynaud described the disease as a neurosis, characterised by an exaggeration of the excito-motor centre in the cord, which presides over the vaso-motor nerves; and he attributed the lozal mischief to cramp-like contractions of the ar terio-capillaries.

In the casa above described, well-marked changes in the peripheral nerves were found on microscopical examination. The spinal cord and nerves were hardened in Müller's fluid and spirit ; sections were made transversely, and stained with eosin and logwood. The spinal cord itself presented but slight changes. The nerve-cells of the anterior cornua were healthy, or nearly so, but those forming the posterior vesicular columns of Clarke presented more or less sphericity of ontline. There was perhaps a little general thickening of the neuroglia. The nerves examined were the following: sciatic, internal popliteal,

${ }^{1}$ Abstract of a paper read at the Pathological Socicty, January 4th, 1887. 